\title{
Interim guidance on the use of the Abbott ID NOW'T instrument and COVID-19 assay
}

\section{on behalf of the Canadian Public Health Laboratory Network and the Canadian Society of Clinical Chemists}

\author{
Suggested citation: Canadian Public Health Laboratory Network and the Canadian Society of Clinical Chemists. \\ Interim guidance on the use of the Abbott ID NOW'M instrument and COVID-19 assay. Can Commun Dis Rep \\ 2020;46(11/12):422-6. https://doi.org/10.14745/ccdr.v46i1112a09 \\ Keywords: guidance, Abbott ID NOW, Canada, public health, COVID-19, point-of-care, SARS-CoV-2
}

This work is licensed under a Creative Commons Attribution 4.0 Internationa License.

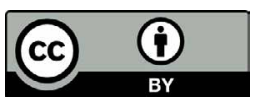

Correspondence:

paul.sandstrom@canada.ca and adrienne.meyers@canada.ca

\section{Introduction}

This document, prepared October 26, 2020, provides interim guidance on the use of the Abbott ID NOW'M instrument and coronavirus disease 2019 (COVID-19) assay in the context of the Canadian public health system.

The Abbott ID NOW COVID-19 assay is an isothermal nucleic acid amplification technology intended for the qualitative detection of nucleic acid from severe acute respiratory syndrome coronavirus 2 (SARS-CoV-2) viral ribonucleic acid (RNA) in direct nasal, nasopharyngeal or throat swabs from individuals who are suspected of COVID-19 by their healthcare provider. While already in widespread use globally, there are several reports of a reduced sensitivity associated with the test when compared with other laboratory-developed tests (LDTs) or commercially available nucleic acid amplification tests (NAAT) such as the Cepheid GeneXpert ${ }^{\mathrm{TM}}$ based assay (1). The use of a lower sensitivity test, even a real-time transcription polymerase chain reaction (RT-PCR) method, carries risks to decision-making that can only be offset by the magnitude of possible benefits. It must be understood that a significantly greater degree of diagnostic uncertainty will be introduced/remain with use of the Abbott ID NOW assay, relative to the conventional RT-PCR methods commonly used in Canada at the time of writing. These guidelines will be updated periodically as more information is available regarding test sensitivity in different settings (surveillance, screening, diagnosis) and in the overall context of infection with SARS-CoV-2.

Many of these guidelines may also be applied to other less sensitive molecular and rapid antigen-based tests that may be approved for use in the future.

\section{Key messages}

- Health Canada provided approval for use of the Abbott ID NOW COVID-19 assay (October 2020).

- The intended use for this assay as outlined by Health Canada is as follows:

- The Abbott ID NOW COVID-19 assay performed on the Abbott ID NOW instrument is a rapid molecular in vitro diagnostic test utilizing an isothermal nucleic acid amplification technology intended for the qualitative detection of nucleic acid from the SARS-CoV-2 viral RNA in direct nasal, nasopharyngeal or throat swabs from individuals who are suspected of COVID-19 by their healthcare provider within the first seven days of the onset of symptoms.

- Results are for the identification of SARS-CoV-2 RNA. The SARS-CoV-2 RNA is generally detectable in respiratory samples during the acute phase of infection. Positive results are indicative of the presence of SARS-CoV-2 RNA; clinical correlation with patient history and other diagnostic information is necessary to determine patient infection status. Positive results do not rule out bacterial infection or co-infection with other viruses.

- Negative results should be treated as preliminary and, if inconsistent with clinical signs and symptoms or necessary for patient management, should be tested with different authorized or cleared molecular tests. Negative results do not preclude SARS-CoV-2 infection and should not be used as the sole basis for patient management decisions. Negative results should be considered in the context of a patient's recent exposures, history and the presence of clinical signs and 
symptoms consistent with COVID-19.

o The Abbott ID NOW COVID-19 test is intended for use by medical professionals or trained operators who are proficient in performing tests using the Abbott ID NOW instrument in laboratory and point-of-care (POC) settings.

- The performance of the assay should be verified in the field before recommending its use. This is critical since data obtained from pre-market evaluations cannot account for the variability in training and quality of sample collection that follows use in a broader population. Therefore, clinical performance must continue to be monitored.

- The "in-field" performance characteristics of the Abbott ID NOW is still under evaluation in Canada; however, data about the nature of the technology from other jurisdictions suggest that the tests have lower sensitivity but comparable specificity to other LDTs and commercial NAAT assays. Although, the rapid nature of the assays makes it suitable for POC applications, these performance characteristics, combined with the incidence of infection within the population being tested, must be considered when interpreting the results.

- In discussion with provincial and territorial laboratory directors, the use of this test must be carefully considered.

- At this time, until further data is collected, because of the decreased sensitivity, all negatives should be considered preliminary negatives.

- Owing to an expected higher rate of false negatives, it is recognized that reflexive laboratory-based testing of preliminary negatives from the Abbott ID NOW COVID-19 testing depending on its proposed use) will likely introduce an additional burden to reference laboratories already facing enormous testing volumes.

- This document outlines scenarios where the Abbott ID NOW COVID-19 testing may prove useful, should the expected performance characteristics be confirmed.

\section{Current approach to severe acute respiratory syndrome coronavirus 2 testing in Canada}

Since the emergence of SARS-CoV-2, testing has been a key pillar of Canada's response to the pandemic. The broad use of testing, as part of an array of public health measures, led to a flattening of the epidemic curve in the spring of 2020, demonstrating the value of testing as a part of the COVID-19 response. To date, testing has relied on molecular (i.e. RT-PCR) testing performed on a nasopharyngeal sample (NP) or alternate respiratory sample collected by a healthcare professional. This testing method currently remains the gold standard for diagnosing SARS-CoV-2 infection in Canada.

\section{Considerations for the use of the Abbott ID NOW COVID-19 assay}

It is critically important to understand the timing of specimen collection in relation to symptom onset since the sensitivity of the test is not expected to be uniform over the course of infection. Data suggest that viral shedding may begin 2-3 days before the symptoms peak-around the time of symptom onset-and then gradually declines over time thereafter $(2,3)$.

During the first seven days of infection, viral loads are likely to be above the limit of detection for the Abbott ID NOW assay. Although the time of post-symptom onset still needs to be carefully considered. It is also important to understand test performance in relation to the time since a potential exposure when the test is being used for rapid contact tracing (e.g. how many days after exposure might one expect to have viral loads that can be optimally detected using the Abbott ID NOW?).

Notwithstanding the difference in the performance profile, other features of the Abbott ID NOW (including but not limited to; faster turnaround time, lower per-test cost, ability to do the test in a setting by non-professionals on a more frequent basis) suggest that it could have an important role to play in the next phase of the pandemic response.

It is important for public health, microbiology and infectious diseases experts to identify the scenarios where the use of the Abbott ID NOW may further strengthen the public health response by expanding access to testing beyond existing indications and increasing capacity for molecular detection of SARS-CoV-2. Furthermore, establishing mechanisms to allow a new $\mathrm{POC}$ test to report into the public health system efficiently is critical (see "Reporting of results and Quality Control" section below).

\section{Balancing test sensitivity against other considerations}

The intrinsic performance characteristics of the Abbott ID NOW are not the only factors determining its utility. The final interpretation of a test must take into account the performance parameters, the prevalence of infection, predictive values and the intended use of the test result. Therefore, the tolerance for sensitivity and specificity thresholds will vary based on the reason for testing and the expected action that would follow either a positive or a negative result. 
In scenarios where critical decisions and actions rely on a test result (e.g. a symptomatic resident in a long-term care home, a patient in an intensive care unit [ICU] who requires immediate treatment), the recommended test would be the most accurate test. At the time of writing, the indicated (best) test would be RT-PCR performed on a NP sample or on a lower respiratory tract sample in patients with evidence of pneumonia. However, there may be circumstances where a rapid POC test can be permissible and enhance testing capacity to support the public health response, particularly when the demand for RT-PCR testing exceeds laboratory capacity or is otherwise unavailable or in situations where a symptomatic individual may otherwise be lost to follow-up.

Clinical situations where infection is prevalent in the community: When the prevalence of infection is high, and the access to RT-PCR testing is unavailable (i.e. northern, remote and isolated [NRI] communities) or when the results are delayed beyond 48 hours because testing demand is exceeding laboratory capacity issues, a POC test may provide an option that will positively impact public health or clinical management. Here the intended use is for early diagnosis of infection. In this situation, a positive result will allow for early implementation of public health measures and contact tracing or clinical management decision-making. Although there will be a need to further evaluate, preliminary data suggests that performance of the Abbott ID NOW in early infection (1-5 days post-symptom onset) may be similar to RT-PCR in terms of sensitivity and performance. However, negative results should be confirmed using conventional NAAT as soon as possible as clinical decisions cannot be made based solely on the Abbott ID NOW test because of the lowered negative predictive value associate with reduced test sensitivity.

Clinical situations where infection is not prevalent in the community and high sensitivity is not the main consideration: There may be alternative settings where a less sensitive test may be acceptable. Although the Abbott ID NOW is currently approved for testing symptomatic individuals within the first seven days of symptom onset, monitoring of asymptomatic individuals who are at risk of introducing infection into high risk settings (e.g. long-term care, correctional facilities) could be considered. Modelling data suggest that testing protocols that incorporate repeated and frequent re-testing of individuals could be effective (4). Here the intended use of a POC test is for monitoring infection in individuals that may not otherwise be able to be tested with the same frequency due to challenges with testing capacity. Due to the potential reduction in pretest probability of a positive result, the test would need to be confirmed using a laboratory-based NAAT. The purpose of this requirement for confirmation is to reduce the potential for negative factors associated with a false positive test (unnecessary removal from work, stigma that may be associated with infection, etc.).

\section{Proposed use scenarios}

At this time, it is not possible to provide an exhaustive list of all cases where the Abbott ID NOW assay might be of benefit. Several scenarios are given as examples below, but are not meant to be proscriptive.

\section{Scenario 1: Northern, remote and isolated settings}

The NRI communities face additional barriers to timely test results due to transportation time required to deliver a specimen to a testing laboratory. Given the importance of accurately identifying new cases in NRI communities to prevent spread of SARS-CoV-2 in the face of limited healthcare resources, RT-PCR testing is the recommended test for these settings. While there have been extraordinary efforts to date to bring high quality POC PCR testing to NRI communities (e.g. Cepheid GeneXpert test), significant challenges remain. First and foremost is the ongoing short supply of the GeneXpert COVID-19 test cartridges. The availability of a relatively low complexity POC solution with an anticipated higher test allocation may be an attractive option as a screen-in test to conserve GeneXpert test cartridges for the testing of symptomatic individuals. In this two-test algorithm, if a sample is shown to be positive using the Abbott ID NOW assay, then appropriate actions can quickly be put in place, while negative results can be confirmed on the highly sensitive Cepheid GeneXpert or in a reference laboratory. This may also be useful in other settings where rapid, accurate results are required (e.g. staging for medical procedures in hospitals involving symptomatic patients). This may have additional benefit in allowing the prioritization of GeneXpert test cartridges for NRI communities.

\section{Scenario 2: Early outbreak identification and investigation}

While the use of a less sensitive test would not be recommended for the exclusive management of an outbreak, testing of symptomatic individuals and their direct contacts with the Abbott ID NOW assay can be useful for the early identification of possible outbreaks.

- $\quad$ Testing can be done as part of suspected outbreak identification and investigation where patients can be tested rapidly on site if faster preliminary results will help inform and expedite public health action (triage of patients and contact tracing). This may be particularly relevant in situations where a symptomatic individual may otherwise be lost to follow-up.

o This would always be followed by confirmatory PCR testing, although this requirement could be revisited to determine if ongoing testing is needed. 


\section{Scenario 3: Asymptomatic testing in high risk settings}

An additional broad category for use of the Abbott ID NOW includes situations that involve the prospective monitoring of asymptomatic individuals for introduction of SARS-CoV-2 into high risk settings. Note that such a proposed monitoring role for non-PCR testing technologies is referred to as "screening" in some other documents on COVID-19 testing strategies. At this time, the market authorization for the Abbott ID NOW from Health Canada-Medical Devices Bureau is focused on symptomatic testing in the early phase of disease, so the use of this test in a monitoring context will require clinical validation. The frequency of repeat testing is not yet defined (see below).

- Repeated testing of workers in congregated settings to prevent introduction or to minimize the chance of spread within a site, including:

- Large processing plants

- Long-term care facilities

- Homeless shelters

o Farm/migrant workers

- Inmates in correctional facilities

- $\quad$ The Abbott ID NOW could be used for prospective testing of low-risk, asymptomatic visitors and staff entering congregated settings.

- Important additional considerations for the use of Abbott ID NOW include the following:

o Testing would always need to be done in the context of PCR confirmatory testing of all positive cases

- Reflexive PCR testing of preliminary negative cases needs to be carefully considered and is not being recommended at this time due to the significant impact it would have on current testing capacity in laboratories already facing enormous testing volumes

o The frequency of repeat testing will need to be carefully examined to ensure the testing strategy can correctly identify individuals during a high viral period early in infection

- The use of alternative specimen collection (rather than with oral or nasal swabs included with the Abbott ID NOW) may be more acceptable for collecting samples from asymptomatic individuals than NPs

These situations represent scenarios where frequent entries and exits multiply the potential introduction of the virus into high risk settings known to facilitate the rapid spread of infection. It is not yet possible to articulate the implementation approach that best supports the public health goals of testing (case identification, isolation contact tracing, etc.). It is clear that a false negative test can occur early in infection even with the most sensitive RT-PCR methods. As such, repeat testing may be necessary to detect infection in cases with high clinical suspicion of disease. The Abbott ID NOW assay may offer ease of use, the ability to conduct testing outside traditional laboratory settings and rapid time to results to enable frequent testing and offset the reduced sensitivity.

\section{Approach to the potential use of the Abbott ID NOW assay}

How the results from the Abbott ID NOW assay are interpreted and how it impacts public health and clinical management of patients need to be considered. To do this, it is critical that end users understand the prevalence of infection in the population they are testing. This necessitates a robust surveillance system that communicates regularly with the end users.

\section{Positive result}

Positive results should be considered "preliminary positive" until confirmed using a reference RT-PCR method. While the Abbott ID NOW assay is expected to have high specificity, false positives can be expected, particularly if the prevalence of infection in the population tested is low, thus decreasing the pre-test probability of the assay. All patients with a positive result will require isolation. If the confirmatory RT-PCR is negative, discontinuation of isolation can be considered depending on the clinical context that generated the initial test.

\section{Negative result}

In interpretation of a negative Abbott ID NOW result, the clinical context of the test (asymptomatic versus symptomatic) and the pre-test probability of infection must be considered. In patients where the pre-test probability of COVID-19 remains high (e.g. known contact, high community transmission), then the individual should undergo confirmatory testing using RT-PCR to direct further management. If the pre-test probability is low, then the individual can be monitored in the absence of isolation and reference testing.

\section{Frequency of testing}

As highlighted above, the Abbott ID NOW assay is ideally used in surveillance/screening programs where individuals get repeat tests to account for a lack in sensitivity. If the Abbott ID NOW is used in a monitoring approach, the ideal frequency of testing remains to be defined. The effectiveness of this strategy is dependent upon several associated factors, including the proportion of asymptomatic infections, the sensitivity of the assay and the time to results (assuming that self-isolation would occur once a positive test is identified).

\section{Reporting of results and quality assurance}

The use of the Abbott ID NOW will most likely occur outside of a laboratory environment. The current anticipated market authorizations are expected to require oversight of the testing 
procedure by a trained healthcare professional, at least in the short term. It will be essential that a mechanism for reporting of results into the public health system and/or laboratory system be developed to ensure appropriate data capture and quality control, and to support public health action.

It is critical that quality assurance practices be considered when implementing POC testing, regardless of the perceived simplicity of the test. Where POC testing is implemented outside a hospital environment, sites are recommended to partner with local accredited laboratories for ongoing guidance and oversight. The laboratory director and partnering laboratories will guide sites to ensure important quality assurance practices are in-place.

Examples of quality assurance practices that must be considered:

- Training and ongoing authorization of staff who will perform POC testing

- Initial and ongoing reagent validation prior to clinical use

- Quality control practices for regular monitoring of test performance

- Proficiency testing to monitor overall testing practices at a site

- Troubleshooting issues with tests and/or devices

- Reporting of results

\section{Critical scientific questions}

The science continues to evolve daily as unprecedented global investment in research and development continues. Despite this, several critical questions remain to inform the use of new tests such as the Abbott ID NOW and sample types.

- How do these tests perform in "real life"?

- Most submissions for approval have used simulated samples to evaluate the tests. This creates uncertainty about the true performance when applied to patients. There must be a verification of performance by comparing the real-life performance of intended use in the field compared with the traditional nucleic acid amplification methodology.

- How frequently is testing required to close the sensitivity gap?

- This requires understanding of the dynamics of the test over time. It will be important to determine the frequency of testing to best mitigate the risk of cases being missed due to the lower sensitivity of the Abbott ID NOW.

- At what threshold of community transmission is repeat testing in specific environments beneficial?
- How do lower sensitivity tests and lower sensitivity sample types interact?

- If the NP swab is considered the gold standard, then what is the impact on sensitivity of using a less sensitive specimen for testing? How do the assays compare when an oral or nasal swab or alternative sample type such as a saline gargle is used?

- If oral and/or nasal swabs are used as an alternative to the NP swab, the impact must be evaluated to inform potential for use.

\section{Competing interests}

None.

\section{Acknowledgements}

The Canadian Public Health Laboratory Network (CPHLN) input is derived from the CPHLN Laboratory Director's Council and Respiratory Virus Infection Working Group (ReVI). We would like to thank members of the CPHLN Secretariat, including A MacKeen and S Radons Arneson for coordination of document synthesis.

\section{Funding}

None.

\section{References}

1. Smithgall MC, Scherberkova I, Whittier S, Green DA. Comparison of Cepheid Xpert Xpress and Abbott ID Now to Roche cobas for the Rapid Detection of SARS-CoV-2. J Clin Virol 2020;128(104428):104428. DOI PubMed

2. Bullard J, Dust K, Funk D, Strong JE, Alexander D, Garnett L, Boodman C, Bello A, Hedley A, Schiffman Z, Doan K, Bastien N, Li Y, Van Caeseele PG, Poliquin G. Predicting infectious SARS-CoV-2 from diagnostic samples. Clin Infect Dis. 2020 May 22:(ciaa638). Online ahead of print. PMID: 32442256 DOI

3. He X, Lau EH, Wu P, Deng X, Wang J, Hao X, Lau YC, Wong JY, Guan Y, Tan X, Mo X, Chen Y, Liao B, Chen W, Hu F, Zhang Q, Zhong M, Wu Y, Zhao L, Zhang F, Cowling BJ, Li F, Leung GM. Temporal dynamics in viral shedding and transmissibility of COVID-19. Nat Med 2020;26(5):672-5. DOI PubMed

4. Mina MJ, Parker R, Larremore DB. Rethinking Covid-19 Test Sensitivity - A Strategy for Containment. N Engl J Med 2020 Sep 30, 2020. DOI PubMed 